\title{
La cuadratura del círculo: el intento de Friedrich von Hayek por resolver la tensión entre igualdad política y desigualdad económica*
}

\author{
Squaring the Circle: Friedrich von Hayek's \\ Attempt to Solve the Tension between Political \\ Equality and Economic Inequality
}

Juan Gabriel Gómez Albarello**

Recibido: 11/12/2013

Aprobado Evaluador Interno: 02/02/2014

Aprobado Evaluador Externo: 20/02/2014

\section{Resumen}

El propósito de este artículo es destacar las contribuciones más importantes que hizo Friedrich von Hayek a la teoría social y política, así como tomar nota de varias críticas dirigidas contra su punto de vista. Este artículo también atribuye el origen de estas contribuciones a la participación de Hayek en el debate acerca de la planificación socialista. La idea más importante de Hayek es que el mercado ofrece la mejor solución al hecho de que el conocimiento social está siempre disperso y es incompleto. En tándem con esta idea, Hayek opuso al modelo de un orden centralizado y planificado el de un orden espontáneo del cual el mercado sería el caso paradigmático. De esta concepción Hayek

\begin{abstract}
The purpose of this article is to highlight the most important contributions Friedrich von $\mathrm{Ha}-$ yek made to social and political theory, and to review various critiques directed against his ideas. This article also traces back those contributions to Hayek's engagement in the debate about socialist planning. Hayek's most important idea is that the market offers the best solution to the fact that social knowledge is always dispersed and incomplete. In tandem with this idea, Hayek opposed to the model of a centralized and planned order another one in which that order is spontaneous, being the market its paradigmatic instance. From this conception, Hayek derived the corollary that the idea of social justice is
\end{abstract}

doi:10.11144/Javeriana.PAPO19-1.ccif

* Artículo de reflexión. El presente texto es el resultado de una investigación acerca del pensamiento político y económico de Friedrich von Hayek.

** Juan Gabriel Gómez Albarello. Profesor Asistente, Instituto de Estudios Políticos y Relaciones Internacionales de la Universidad Nacional de Colombia. Abogado, Universidad Externado de Colombia (1995); PhD en Ciencia Política, Washington University en St Louis (2006). Líneas de investigación: teoría política y relaciones internacionales. Correo electrónico: jggomeza@unal.edu.co. 


\section{Juan Gabriel Gómez Albarello}

derivó el corolario de que la idea de justicia social es un espejismo puesto que, en una sociedad compleja, la posición y el ingreso de cada uno depende de incontables interacciones. Siguiendo esta línea de razonamiento, Hayek reforzó su convicción según la cual la intervención de los gobiernos democráticos en el mercado debería ser limitada severamente. Desde la izquierda y la derecha, varios críticos han mostrado fallas en los argumentos de Hayek que conciernen al carácter disperso e incompleto del conocimiento social, la relevancia de la justicia social, la interdependencia entre el Estado de derecho y la democracia, así como la idea de que el mercado no es un orden que se auto-genera sino que depende de un amplio conjunto de instituciones sociales.

\section{Palabras clave:}

Hayek; conocimiento incompleto; orden espontáneo; mercado; justicia social; Estado de derecho; democracia a mirage for, in a complex society, each one's position and income is the result of uncountable interactions. Following this line of reasoning, Hayek strengthened his conviction that democratic governments' intervention in the market should be severely limited. From left and from right, various critics have shown shortcomings in Hayek's arguments that concern the idea of incomplete and dispersed social knowledge, the relevance of social justice, the interdependence between the rule of law and democracy and the idea that the market is not a self-generating order but rather depends on a larger set of social institutions.

\section{Keywords:}

Hayek; incomplete knowledge; spontaneous order; market; social justice; rule of law; democracy 


\section{La Cuadratura del Círculo: El Intento de Friedrich von Hayek por Resolver la Tensión entre Igualdad Política y Desigualdad Económica}

La tensión entre igualdad política y desigualdad económica es un problema central de la teoría política contemporánea. Esto no quiere decir que no haya otros problemas importantes, siendo uno extremadamente importante el de la relación sociedad-naturaleza. El carácter central de la tensión entre igualdad política y desigualdad económica como problema de la teoría política significa meramente que buena parte de los asuntos que nos conciernen como ciudadanos remiten a él. Si ya hubiésemos resuelto ese problema podríamos dedicarnos más tranquilamente a otras cosas. Pero no podemos. La razón es que el mundo moderno sigue siendo un problema sin solución debido a que, entre otras cosas, nuestras instituciones están basadas en principios contradictorios. Para poder funcionar, la democracia precisa de la reafirmación constante de la igualdad política. Sin embargo, el mecanismo fundamental de intercambio en nuestras economías es el mercado, un mecanismo que, de suyo, genera desigualdades.

Para resolver la tensión entre igualdad política y desigualdad económica, al modo de otros intentos hechos en el pasado, podríamos mercantilizar la democracia o democratizar el capitalismo. En el primer caso, podríamos aplicar de forma irrestricta en la esfera política algunos de los principios que rigen actualmente en la esfera de la actividad económica. Por ejemplo, podríamos abolir las leyes que regulan las campañas electorales con el argumento de que interfieren con la libre competencia política y suprimir nuestros escrúpulos acerca de la forma en que grupos de interés controlan el flujo de información en los medios. En tal caso, trataríamos la política democrática como un mercado en el cual los ciudadanos seríamos consumidores de propuestas de políticas públicas, los partidos políticos sus productores (o propagadores) y los medios empresas encargadas de propagar información sin mayores restricciones acerca de su imparcialidad y su confiabilidad. De hecho, hay toda una tradición en la ciencia política que concibe la democracia justamente como un mercado, como por ejemplo plantean Buchanan y Tullock (1962), Down (1957), Riker (1982) y Wittman (1995).

En el segundo caso, el de la democratización del capitalismo, podríamos forzar una distribución igualitaria de todos los recursos de producción e instituir una agencia encargada de mantener esa distribución igualitaria (Lenin, 1993). Así como cada ciudadano tiene un voto y nada más que un voto, cada uno tendría cierta suma de recursos y nada más que esta suma. Si escogiéramos esta solución terminaríamos por abolir el mercado. La agencia creada para mantener la igualdad intervendría permanentemente en la esfera de las decisiones económicas al costo de restringir en un alto grado, si no abolir, toda iniciativa individual (Friedman y Friedman, 1980).

También podríamos encontrar una manera de sobrellevar la tensión entre igualdad política y desigualdad económica considerándola insoluble, pero haciendo al mismo 
tiempo una apuesta contra el despotismo. Tal es la solución que ofrece la obra de Friedrich von Hayek. Conocer esta obra es participar en un diálogo acerca de nuestro mundo moderno y sus profundas contradicciones. Sin embargo, para incorporarnos a esta conversación es necesario que levantemos la mirada por encima de nuestros prejuicios. La obra de Hayek nos espera más allá de la pila de etiquetas bajo la cual ha quedado sepultada.

Junto con Milton Friedman, Friedrich von Hayek es considerado uno de los padres fundadores de la doctrina neoliberal (Stedman, 2012). Por esta vía, desafortunadamente, su nombre ha quedado asociado al dogmatismo de la desregulación financiera y la economía de casino, a la privatización de todo lo que existe en el mundo (salud, educación, agua, etc.), a la imposición de políticas restrictivas de ajuste por parte de instituciones económicas internacionales (el Banco Mundial y el Fondo Monetario Internacional) sin ninguna consideración acerca del efecto que esas políticas tienen en el funcionamiento de la democracia, así como a la operación de grandes empresas trasnacionales que defienden el capitalismo pero que aborrecen el mercado. Bien vale la pena preguntarse si Hayek habría estado de acuerdo con todo esto. Si traspasamos las cumbres neoliberales podremos llegar a la región de las contribuciones que Friedrich von Hayek hizo a la teoría social y a la filosofía política que han sido fundamentales; sin ellas nuestra comprensión del mundo sería mucho más pobre de lo que ya es.

Antes de realizar una valoración de su obra, quisiera hacer un encomio del hombre. Quisiera resaltar dos virtudes de Hayek que nos sirven de modelo: su anti-conformismo y su humildad. Cuando el acuerdo más extendido entre intelectuales y políticos era el de avanzar hacia una creciente estatización de la actividad social, Hayek tuvo el valor de ir contra-corriente. En 1944 publicó Camino de Servidumbre, y desde entonces promovió la discusión de sus ideas anti-estatalizantes. El celo con el cual Hayek defendió la libertad individual en el ámbito de la filosofía política y la teoría social tiene un paralelo en la literatura: el de la lucha de George Orwell contra el totalitarismo. Creo que no es una vana coincidencia que Orwell haya concebido su célebre novela 1984 justo el mismo año en el cual Hayek entregó a la imprenta su crítica del estatismo.

30 años después, junto con Gunnar Myrdal, Hayek recibió el Premio Nobel de Economía "por su trabajo pionero en la teoría del dinero y de las fluctuaciones económicas, y por su penetrante análisis acerca de los fenómenos económicos, sociales e institucionales”" En el discurso que pronunció con ocasión del Banquete ofrecido en su honor, Hayek propuso que los recipientes de ese Premio tuvieran que realizar un juramento de humildad, similar al juramento hipocrático, consistente en nunca exceder los límites de su competencia en pronunciamientos públicos. Hayek (1974) temía, con razón, que el Premio Nobel le

\footnotetext{
${ }^{1}$ Comunicado de prensa del Comité del Premio Nobel de Economía. Disponible en http://www. nobelprize.org/nobel_prizes/economic-sciences/laureates/1974/press.html
} 
otorgaría a sus recipientes una influencia no merecida sobre los legos en materias económicas. No se equivocaba, puesto que la prensa tiende a tratar a estas personas como omnisapientes, sus opiniones sobre los más diversos temas son tratadas con una reverencia que está fuera de lugar (basta con pensar, por ejemplo, en Paul Krugman).

No es usual encontrar en la academia expresiones semejantes de humildad. En el caso de Hayek, su modestia proviene de sus más profundas convicciones acerca del conocimiento humano. Hayek (1952, 1960, 1967, 1975) desconfió de nuestra capacidad para descifrar el orden que tienen las cosas; creía que todas nuestras aproximaciones eran siempre imperfectas y que, por lo tanto, no deberíamos presumir mucho de ellas. Corolario de lo anterior es la proposición según la cual el vano orgullo de creer que se sabe cómo funcionan las cosas y cómo se podría hacerlas funcionar mejor ha nutrido la arrogancia de muchos déspotas y ha sido la fuente de innumerables abusos (Hayek, 1960).

Dicho esto acerca del hombre, quisiera presentar la obra de Hayek del siguiente modo: en primer lugar, voy a referirme a la que considero es su contribución central al pensamiento social, económico y político, esto es, su argumento acerca del carácter incompleto y disperso del conocimiento que tenemos los individuos en la sociedad, haciendo un especial énfasis en el contexto en el cual Hayek elaboró ese argumento. Luego, en una segunda parte, presentarélas principales implicaciones que el mismo Hayek extrajo de su planteamiento y que se enlazan con su preocupación acerca del despotismo político, una preocupación que está presente en casi todas las páginas que escribió. Finalmente, presentaré varias críticas que Hayek ha recibido desde la izquierda y desde la derecha.

\section{El argumento epistemológico contra el socialismo: origen y significado de la principal contribución de Friedrich von Hayek al pensamiento económico y político}

A comienzos del Siglo XX hubo un debate muy importante acerca de las posibilidades y límites de la planificación socialista. Ese fue un debate motivado, en parte, por la crisis más profunda que ha tenido hasta ahora el capitalismo y por la victoria de los Bolcheviques en Rusia. Los Bolcheviques, luego de varias dificultades para reactivar y estabilizar la economía rusa, se decidieron por un enfoque de economía planificada, el cual definió el modelo de desarrollo de la entonces recién creada Unión Soviética. Casi al mismo tiempo que los soviéticos habían comenzado un proceso de industrialización acelerada de su país, en los países capitalistas occidentales el funcionamiento de la industria y de todos los demás sectores de la economía se había venido a pique. Luego del pánico desatado en la Bolsa de Nueva York a finales del mes de octubre de 1929 se evaporó la confianza de muchos ahorradores e inversionistas, lo que produjo una depresión económica bastante severa. Los precios de todos los productos cayeron y mucha gente entonces perdió su empleo. Aunque las bodegas estaban llenas de cosas, gente sin trabajo carecía del dinero para poder comprarlas. Sin trabajo y sin comida, 


\section{Juan Gabriel Gómez Albarello}

lo que mucha gente pensaba era que el sistema económico que había producido esta situación tenía que ser reemplazado por uno mejor, uno en el cual un grupo de expertos planificadores hiciera el trabajo que el mercado había dejado de hacer.

Los economistas marxistas habían denunciado el capitalismo como una economía anárquica, incapaz para resolver las crisis que él mismo generaba (Engels, 1977). A la imagen de la desocupación y el paro en las sociedades occidentales, estos economistas contrapusieron la de la rápida industrialización de la Unión Soviética (Bukharin, 1982; Dobb, 1928). En su opinión, el socialismo en su versión de economía centralmente planificada, era superior al capitalismo (Neurath, 2004). ${ }^{2}$

Ludwig von Mises (1951), economista austríaco y mentor intelectual de Hayek, replicó que el socialismo era en realidad la planificación del caos, un pobre sustituto de la anarquía del mercado. Según Mises, el socialismo no podía ser eficiente por carecer del sistema de comunicación que permite la adecuada asignación de recursos en la sociedad: el sistema de precios. El sistema de precios es un sistema de comunicación puesto que cada precio es una señal que envía un productor acerca de cuánto está dispuesto a recibir por lo que ha producido; con cada compra, quien adquiere un bien o un servicio comunica cuánto valora lo comprado, transmite una pieza de información consistente en indicar lo que para esa persona representa el bien o servicio adquirido. Para realizar una transacción, un productor y un comprador no tienen que preguntar necesariamente qué es lo que hacen otros productores y otros consumidores. Cada quien formula una elección con base en el conocimiento que tiene de la oferta y la demanda en cada caso. Así las cosas, el sistema de precios es un sistema descentralizado que funciona en una escala muy grande y, de este modo, permite que las decisiones anónimas que toman muchos individuos puedan coordinarse sin la intervención de una agencia ajena a esos individuos.

Los economistas marxistas respondieron a Mises argumentando que el socialismo sí podía servirse del sistema de precios. Oscar Lange (1936 y 1937) y Abba Lerner (1934, 1936 y 1937) sostuvieron que era posible asignarle precios a los bienes de consumo y a la fuerza de trabajo utilizando como referencia los costos de producción de las empresas. De esa forma sería posible determinar en forma óptima lo que las empresas deberían producir y en qué cantidad, los precios seguirían funcionando como un sistema de señales. Sin embargo, este sistema no estaría distorsionado por el afán de lucro propio de los productores de un sistema capitalista. Hayek (1948) intervino en el debate destacando varios supuestos cuya observancia era necesaria para que el sistema de planificación y

\footnotetext{
${ }^{2}$ No todos los marxistas fueron entusiastas con respecto a la capacidad de ordenar y dirigir la economía mediante la planeación centralizada. Un importante marxista crítico de este modelo, cuya obra leyó Hayek, fue Maurice Dobb. Varios de sus ensayos publicados en los años 1930s fueron recogidos y publicados en 1955 en el volumen On Economic Theory and Socialism.
} 
precios funcionara. ${ }^{3}$ El primero de estos era la existencia de un mecanismo de coordinación entre las empresas que permitiera alinear, por así decirlo, los costos de producción, el precio de la fuerza de trabajo y el precio de los bienes de consumo. Hayek sostuvo que ese mecanismo existía en el mercado. En ausencia del mercado tendría que haber una gran junta en la que tuviesen asiento todas las empresas, que se dedicara a asignar precios según la demanda de bienes y el volumen de la fuerza de trabajo y del capital existente.

Hayek puso en cuestión la eficiencia de este mecanismo al señalar que el modelo de planificación y precios era estático. El modelo podría funcionar siempre y cuando hubiese un conocimiento completo de cada uno de los elementos de la ecuación con la cual se establecieran los precios. Si el grado de insatisfacción de las necesidades fuese siempre igual, si hubiese un mismo nivel de productividad dada la cantidad existente de fuerza de trabajo y de capital, el modelo de planificación y precios funcionaría. Sin embargo, la realidad es bien diferente. Una vez que están satisfechas ciertas necesidades, los deseos de consumo de la gente se orientan hacia otros objetos. La productividad puede aumentar gracias a cambios tecnológicos o puede disminuir debido a factores que no están bajo el control de los planificadores. En suma, un modelo estático de asignación de recursos mediante los mecanismos de planificación y precios difícilmente podría resolver los problemas de una economía dinámica. Al estar limitada su capacidad de adaptación a cambios en el entorno, cambios no controlados por los planificadores, los mecanismos de coordinación dejarían de ser eficientes. La planificación, en vez de funcionar mejor que el mercado, lo haría peor.

En el contexto de esta discusión Hayek hizo su contribución más original. En un artículo publicado en el American Economic Review en 1945, Hayek sostuvo que el mercado es un mecanismo de coordinación de la acción de muchos individuos que funciona por la vía de permitir la circulación de un conocimiento desagregado, particular, dependiente de la perspectiva y la posición de cada individuo en la sociedad. De este planteamiento se sigue que ningún burócrata, ningún planificador, tiene un conocimiento adecuado de cuáles son las necesidades y preferencias de cada individuo. A ningún burócrata le será posible determinar cuánto valor le asigna cada individuo a cada objeto de consumo, dadas esas necesidades y esas preferencias. Lo más que podría hacer sería postular un modelo universal de necesidades por satisfacer que haría abstracción de las diferencias existentes entre todos los individuos. Este sistema, por lo tanto, carecería de la capacidad para integrar en su funcionamiento el conocimiento específico que cada individuo tiene de lo que necesita y de lo que quiere.

Lo dicho anteriormente no niega que todos los individuos tengamos necesidades similares. Todos necesitamos comida y descanso, educación y salud, vivienda y

\footnotetext{
${ }^{3}$ Hayek articuló este punto de vista en varios ensayos publicados durante la segunda mitad de los años 1930s y 1940s, los cuales fueron reunidos en el volumen publicado en 1948 bajo el título Individualism and Economic Order.
} 


\section{Juan Gabriel Gómez Albarello}

esparcimiento, etc. Empero, cada individuo sabe qué es lo que tiene y qué es lo que puede dar en contraprestación por las cosas que quiere y a las cuales les ha atribuido valor. Es cada individuo quien conoce, sin que necesariamente la tenga que conocer los demás, la cantidad apropiada de cada una de esas cosas que le dejarían satisfecho. Es también él o ella quien sabe cuánto está dispuesto a dar a cambio por todas esas cosas. Un sistema que agregara ese conocimiento en una instancia central para determinar la cantidad óptima que se debe producir de cada cosa truncaría la circulación del conocimiento que tiene cada individuo, no sólo de sus preferencias sino también de las cosas que usa para satisfacerlas. Lo que de ese modo se trunca no es meramente lo que cada individuo conoce del mundo y de sí mismo. Lo que se atrofia de este modo es la capacidad de adaptación social que surge del delicado balance que realizamos entre lo que tenemos, lo que queremos y lo que estamos dispuestos a dar por ello, dadas las oportunidades que tenemos para intercambiar una cosa por otra.

Del apremio por superar las limitaciones de ese balance, surge a su vez el ingenio y la inventiva que permite que, beneficiándome yo de una nueva forma de hacer las cosas, otros también lo hagan. Al competir con otros tengo el incentivo para usar mi ingenio, lo cual es el argumento clásico a favor de la mayor eficiencia del mercado en la asignación de recursos. Pero no es solamente el ingenio lo que pongo a circular en la trama de las transacciones en la que participamos todos los individuos. Pongo a circular también un conocimiento particular del cual toman nota otros individuos y que les sirve para ajustar la medida de lo que tienen, de lo que quieren y de lo que a su vez ellos están dispuestos a dar.

Este argumento epistemológico de Hayek fue su contribución decisiva al entendimiento de la forma como funcionan las instituciones económicas de una sociedad compleja. Es un argumento epistemológico porque se refiere a la forma como los individuos usamos un conocimiento desagregado, particular cuando participamos en la cadena extensa, compleja y anónima de intercambios económicos. ${ }^{4}$

Hayek cree que en sociedades complejas no hay un sustituto del mercado que sea más eficiente, ni siquiera uno que sea plausible. El trueque es un sistema de intercambio imposible en una sociedad en la cual las preferencias de los individuos son muy diversas y en la cual la suma de los objetos de esas preferencias es muy grande. Tampoco es posible que un sistema de redistribución operado por una instancia central funcione de forma óptima. De partida, el problema computacional que tendría que resolver sería de una extraordinaria complejidad. Esto suponiendo que todos los individuos proporcionaran información confiable acerca de sus necesidades y sus capacidades. El tema no es sólo la complejidad de

\footnotetext{
${ }^{4}$ Hayek refinó estos planteamientos a lo largo de su vida. Su formulación más acabada se encuentra en el volumen que publicó en 1973: Law, Legislation and Liberty: A New Statement of the Liberal Principles of Justice and Political Economy, 1: Rules and Order. Londres: Routledge \& Kegan Paul.
} 
agregar la información y de construir una función de asignación de recursos que fuese eficiente, asegurando que cada individuo recibiera una canasta de bienes y servicios correspondiente a sus necesidades. Incluso si ese arduo problema computacional pudiera ser resuelto, el incentivo que tendría cada individuo para distorsionar el conocimiento que tiene de sus necesidades haría imposible que la agregación centralizada del conocimiento de todos sirviera para una asignación eficaz de los recursos a cada uno.

Pensemos por un momento en lo que sería vivir en el hipotético mundo en el cual una agencia central, con base en un plan, tuviese a cargo redistribuir los recursos existentes en la sociedad. Detengámonos a considerar el hipotético proceso de agregación de conocimiento que tendría que llevar a cabo esa agencia central. Es muy posible que cada individuo exagerara la cantidad de tiempo que tendría que emplear para realizar sus tareas y que disminuyea el valor de los bienes y servicios que recibiera a cambio de su trabajo. Por un lado, exagerar el tiempo necesario para realizar su trabajo le permitiría contar con más tiempo que podría dedicarle a otras actividades; por el otro, disminuir el valor de lo que recibiría a cambio le podría dar acceso a más cosas que, de otro modo, estarían fuera de su alcance.

Uno podría objetar que estas distorsiones podrían ser corregidas estableciendo un mecanismo de revisión de los reportes de cada individuo. Sin embargo, este mecanismo tendría que operar conjuntamente con un sistema de verificación y vigilancia que supondría que todas las actividades estuviesen siempre bajo la mirada del Estado. Al establecer dicho sistema, la discreción de los funcionarios encargados de esa verificación y vigilancia sería muy grande; fácilmente podría degenerar en la arbitrariedad. A su turno, cada individuo tendría incentivos para negociar con los funcionarios la forma en la cual ejercieran su poder discrecional. Es decir, a cambio de un favor particular al funcionario, uno podría obtener otro favor particular: un reporte del valor del trabajo por uno realizado o un reporte de las cosas que uno necesitara sesgado a favor de la obtención de más cosas de las que uno debería obtener si el reporte de ese funcionario fuera honesto.

Este escenario hipotético no lo es tanto. El sistema de planificación y precios de los países socialistas funcionó de forma paralela con un mercado de favores entre funcionarios y ciudadanos por fuera de ese sistema. Además, funcionó de forma paralela con otro mercado, un mercado de verdad en el que la gente intercambiaba cosas y servicios de un modo no previsto y no controlado por el Estado. A falta de mercado la gente creó uno, pero un mercado negro que operó con todas las distorsiones características de toda economía subterránea (Dowlah y Elliot, 1997; Hanson, 2003).

No hay que ir tan lejos. Nos bastaría recordar cómo eran las cosas cuando la empresa estatal de teléfonos no tenía competencia, esto es, cuando no había un mercado de las telecomunicaciones. Lograr que a uno le dieran una línea telefónica era una proeza. Usualmente era necesario hacer una larga fila para formular el pedido de instalación de un teléfono y eso no era suficiente. Muchas veces la asignación de una línea se obtenía recurriendo a 
algún tipo de influencia sobre los funcionarios encargados de tomar la decisión. Ahora las cosas son diferentes. Si bien es cierto que las empresas de telefonía no prestan su servicio en las mejores condiciones y que es necesario muchas veces hacer reclamaciones acerca de fallas y cargos injustos, la obtención de una línea telefónica se ha convertido en un proceso mucho más simple. Hay un mercado en el cual la oferta responde mejor a la demanda que lo que lo hacía el Estado en la época en la cual no existía ese mercado.

Con esto no quiero decir que la mejor oferta en salud y en educación se lograría privatizando la prestación de estos servicios públicos. Antes bien, creo que por razones de equidad e inclusión social y económica, la oferta de estos servicios debe ser mayormente pública; incluso por razones de eficiencia ese servicio debe ser público. Estados Unidos es el país rico que más gasta en salud, y no por ello tiene el mejor sistema. Antes bien, con un esquema de prestadores privados, su nivel de cobertura y atención es peor que el de muchos países en los cuales el servicio de salud es pública (Davis, K. et al, 2007). Dicho esto, sí creo que hay muchas otras áreas de la actividad económica en las cuales nos beneficiaríamos de la existencia de más y no de menos mercado.

Al formular su argumento epistemológico, Hayek no se detuvo a considerar un aspecto que hoy es decisivo para comprender la forma en que se realizan los intercambios de bienes y servicios en el mercado: el efecto que tiene sobre esos intercambios la manipulación de la moneda que continuamentellevan a cabo los Estados así como otros agentes económicos. En el mercado la moneda es una herramienta básica; si no existiera, nuestros intercambios se limitarían al trueque. Precisamos del dinero para tomar parte de la cadena extensa, anónimay compleja de intercambios que tienen lugar en el mercado. Sin embargo, esta herramienta no es neutral. Los Estados se han arrogado el privilegio de ser los únicos con autoridad para emitir moneda y muchas veces abusan de ese poder. Al hacerlo, distorsionan el sistema de comunicación mediante el cual los individuos coordinan sus acciones en el mercado.

Cuando Hayek tomó nota de esta situación propuso que los Estados renunciaran a su poder de emitir moneda. Creyó necesario devolverle al mercado este poder con el fin de restablecer una disciplina que muchos Estados no están dispuestos a observar (Hayek, 1976b). Ésta no es, desde luego, la única alternativa. El planteamiento de Silvio Gesell (1958) acerca del modo en que debería producirse y circular la moneda es, a mi juicio, digno de toda nuestra atención. Gesell vio claramente que el modo actual de producción y circulación del dinero favorece a los bancos y que, con la introducción de un mecanismo que les diera a los individuos un incentivo para hacerlo circular rápidamente, la posición dominante de los bancos desaparecería.

\section{Las implicaciones políticas y sociales del argumento epistemológico en favor del mercado}

En su opúsculo La Revolución Rusa, Rosa Luxemburg (1969) vio con clarividencia que donde se destruye la libertad política lo único que queda en pie es la burocracia. El 
fracaso del modelo soviético demostró cuán acertada fue su predicción. Este fracaso motivó a muchos marxistas, incluso antes de la caída del Muro de Berlín, a revisar sus planteamientos acerca de la democracia (Poulantzas, 1979), pero han sido mucho más reticentes a revisar sus creencias acerca del mercado. Sería muy saludable tomaran nota de los planteamientos de Hayek pues él vio con clarividencia que donde se destruye el mercado también se destruye la libertad política.

El marxismo fue el gran heredero de la fe en la razón propia de la Ilustración. Motivados por esta fe, muchos marxistas creyeron que era posible reordenar la sociedad, reorganizarla, reconstruirla sobre bases eminentemente racionales. ${ }^{5}$ De su interpretación racionalista de la historia de la humanidad extrajeron la conclusión de que los seres humanos podían romper las ataduras del mundo heredado de las generaciones anteriores, mantenidas gracias a la fuerza del hábito y protegidas por un sistema supersticioso de creencias. El gran disolvente de esas ataduras fue el capitalismo. El poder corrosivo del mercado erosionó las instituciones de la sociedad tradicional, pero mantuvo dividida a la humanidad entre quienes se apropiaban de la riqueza generada por el trabajo y quienes tenían que vender su trabajo para generar riqueza. Si la generación de riqueza fuese controlada por toda la sociedad, entonces la sociedad podría superar la división que motiva continuamente una lucha intestina en su seno. Para establecer ese control y ordenar racionalmente la producción y distribución social de la riqueza, el marxismo postuló que era preciso abolir la propiedad privada sobre los medios de producción y, consiguientemente, el mercado (Marx, 1998).

Hayek contrapuso al modelo de construcción racional del orden social la idea del surgimiento espontáneo del orden. Al plan deliberado por establecer un orden en la sociedad, Hayek opuso el modelo de un orden que surge gracias a las acciones de los individuos, pero sin que esos individuos actúen motivados por la realización de un único plan. ${ }^{6}$ Según Hayek, muchas instituciones sociales han sido el fruto de un aprendizaje adquirido mediante el ensayo y error de muchos individuos. En efecto, hay muchas reglas que han surgido de un proceso anónimo de ajuste y adaptación. En tales casos, cuando los individuos resuelven un conflicto o un problema de coordinación, despliegan y revisan continuamente el conocimiento incompleto y disperso que tienen acerca de la forma de hacer las cosas.7 Por esta razón, cada institución surgida de forma espontánea

\footnotetext{
${ }^{5}$ Lo anterior no significa que no hubiese discontinuidades y rupturas, mas hay importantes líneas de continuidad siendo la más importante la mencionada fe la capacidad racional de la especie humana (Hook, 1968; Kolakowski, 1978).

${ }^{6}$ Tal y como lo ha destacado Christina Petsoulas (2001), Hayek es a este respecto deudor de la Ilustración escocesa.

7 Esta contribución de Hayek ha tenido una profunda influencia en la teoría económica contemporánea. Oliver Williamson (2000) ha indicado explícitamente que la obra de Hayek fue decisiva
} 
refleja el proceso idiosincrático de adaptación a las circunstancias particulares en las cuales se encuentra cada individuo.

La creación de instituciones con arreglo a un plan deliberado tiende a hacer caso omiso de esas diferentes circunstancias o, por lo menos, a considerar que las únicas circunstancias relevantes son las que han sido tomadas en cuenta en ese plan. Hayek siempre sospechó de la posibilidad de reordenar la sociedad de ese modo. La posibilidad de darle una forma distinta a la sociedad de acuerdo con un plan deliberado presupone un conocimiento preciso acerca de su funcionamiento. Si uno postula, por el contrario, que una agencia central no puede agregar ese conocimiento, entonces el fundamento de tales planes se cae por su propio peso. Habremos de considerar que planes de ese tipo son una desmesura de la razón y un intento por justificar el poder de quienes se atribuyen la capacidad de reformar la sociedad. Contra quienes se arrogan el título de arquitectos de un nuevo orden social, hemos de elevar la sospecha de que lo más que podrán hacer, incluso si están motivados por intenciones altruistas, es instaurar un nuevo despotismo.

Hayek vio en el mercado un ejemplo paradigmático de un orden espontáneo (el lenguaje es otro ejemplo de este orden). ${ }^{8}$ El surgimiento del mercado en la Edad Media se encuadra bien en el modelo de Hayek puesto que en esa época las instituciones que gobernaban el comercio fueron creadas y puestas en vigor por los mismos comerciantes. Aunque es cierto que el derecho romano tuvo una influencia decisiva en todo este proceso, es preciso destacar que los comerciantes carecían del recurso a una autoridad estatal que hiciera obligatorio el cumplimiento de las reglas aplicables en el curso de sus intercambios. No había códigos promulgados por los Estados sino instituciones continuamente adaptadas y ajustadas a las necesidades de la práctica comercial. La doctrina del libre comercio, elaborada en reacción contra las políticas mercantiles de los Estados modernos surgidos en Europa, puede ser vista dentro de este mismo marco de interpretación como un intento por devolverle a la sociedad el dinamismo perdido con el establecimiento de monopolios y reglas destinadas a proteger la producción nacional frente a la extranjera.

Con el fin de resaltar la coincidencia entre la libertad política y la libertad económica, en su obra Hayek soslaya numerosos aspectos de la puesta en vigor de la doctrina del libre

para revisar los supuestos del modelo neoclásico y tomar nota de la interacción entre órdenes formales e informales, así como del efecto de la incertidumbre y del conocimiento incompleto de los individuos. Otro tanto ha hecho Douglas North (2004). Por su parte, Vernon Smith (2005) ha destacado que su programa de investigación en economía experimental acerca de la forma como los individuos usan su conocimiento es deudor de la obra de Hayek.

${ }^{8}$ Hayek recurrió al término griego catalaxia para referirse al orden emergente en una economía de mercado no de acuerdo con un plan deliberado sino como resultado de la interacción de diferentes individuos con creencias, preferencias y recursos distintos. El punto fundamental con respecto a este tipo de orden es que proporciona la solución óptima en un mundo en el cual prevalece la incertidumbre y cada quien sólo puede hacer uso del conocimiento incompleto que tiene. 
comercio. A este respecto, el marxismo proporciona una descripción y explicación más precisas al destacar el papel jugado por los Estados modernos tanto en el orden interno como en el externo: el cercado de tierras que fueron previamente de uso comunal y la expropiación de los campesinos de su trabajo fueron medidas que Estados modernos como el británico y el francés pusieron en vigor de modo concurrente con políticas coloniales de extracción y saqueo de la riqueza en sus territorios de ultramar (Marx, 2000). ${ }^{9}$ La continuación de políticas extractivas mediante el uso de la violencia por parte del Estado, así como por parte de grupos para-estatales, pone de presente que hay muchos lugares en el mundo en los cuales el mercado no tiene nada de espontáneo (Brass, 2011).

No obstante, la distinción que hace Hayek entre un orden que surge de la interacción espontánea de los individuos y un orden planificado desde arriba captura un aspecto muy importante de lo que significa ser libre. En una sociedad en la cual todo es planificado desde arriba la libertad no existe; en una sociedad con un orden espontáneo la actividad libre de los individuos da origen al orden y se sirve de ese orden para continuar existiendo. El siguiente cuadro es útil para ilustrar este contraste. En una sociedad planificada hay un gran número de oficiales que intervienen en el tráfico diciéndole a los individuos a dónde pueden ir y cómo se pueden transportar; en una sociedad con un orden espontáneo hay señales de tránsito que les indican cuándo deben detenerse y cuándo pueden ponerse en marcha, pero no hay agentes públicos diciéndole a la gente a dónde puede ir, mucho menos a dónde tiene que ir. Justamente, un rasgo característico de sociedades en las cuales la libertad se ha extinguido es que los individuos carecen de derechos: sólo conocen prohibiciones y órdenes emitidas por los agentes del Estado.

Aquí quisiera resaltar un aspecto importante de la idea de libertad de Hayek. Hayek no concebía la libertad como ausencia de orden. Antes bien, creía que el orden era esencial a la libre acción individual. Donde los individuos carecen de reglas que les permitan coordinar sus acciones y resolver sus conflictos, la libertad termina consumida por el desorden y luego por la tiranía, pues esta última surge muchas veces como reacción a la percibida falta de orden. Su idea de libertad era negativa, conforme a una respetable tradición liberal. Lo negativo hace alusión aquí a la negación, a la ausencia de restricciones exteriores. Contrariamente a la idea republicana, positiva de la libertad como autolegislación, esto es, como la capacidad de cada quien para darse a sí mismo las leyes que gobiernan sus acciones, Hayek concebía la libertad meramente como la posibilidad de escoger un curso de acción u otro libre de la interferencia de agentes externos.

En este mismo orden de ideas, vale la pena destacar que Hayek no confiaba mucho en la democracia sino en el Estado de derecho, en el imperio de la ley. Como muchos otros liberales antes que él, para Hayek la pregunta acerca de los límites del gobierno

\footnotetext{
${ }^{9}$ Cercano a este análisis es la obra de sociología histórica de Karl Polanyi (2001).
} 
era más importante que la pregunta acerca del titular del gobierno. Para un demócrata, las cosas son al contrario. Lo decisivo es que gobierne el pueblo y que lo haga mediante el uso de una regla que es sinónimo de la democracia: la de la mayoría (Bobbio, 1988). Para Hayek, la regla de la mayoría podía ser un sinónimo de opresión. No importa que sea uno o muchos quienes opriman: opresión siempre es opresión.

El punto de Hayek es que el proceso democrático permite que diferentes grupos de interés formen coaliciones para oprimir a otros, que se sirvan de la regla de la mayoría para favorecer sus intereses. Estos grupos nunca actúan motivados por el interés general, siempre lo hacen por un interés particular. El proceso de deliberación democrática no sirve de freno al deseo de estos grupos de ver sus intereses resguardados por la legislación. Antes bien, se ha convertido en una farsa en la cual la ley ya no proporciona una protección igual sino que es usada como un instrumento para imponer restricciones de entrada al mercado con el fin de capturar rentas, o como un medio de otorgar subsidios y otros beneficios particulares a ciertos grupos (Hayek, 1979). Es difícil controvertir esta descripción del proceso democrático. Las cosas se parecen más a como Hayek las plantea que a las versiones idealizadas del proceso democrático en las diferentes versiones del modelo de democracia deliberativa.

Para Hayek, la solución consiste en limitar la acción del Estado. Puesto que la reducción de la ley a instrumento de intervención en la economía es lo que proporciona el incentivo a grupos de interés para hacer reglas a su medida, es mediante la limitación del poder del Estado como se podrá evitar esta distorsión de la ley. De ahí su fuerte énfasis en el Estado de derecho más que en la democracia. Hayek, sin embargo, soslayó la forma en la cual la concentración de poder económico distorsiona los intercambios que se realizan en el mercado y, consiguientemente, dejó de considerar el papel positivo que juega la ley al darle una protección especial a quienes están en una situación desventajosa. Justamente, una de las premisas del Estado social de derecho es que no debe haber un trato igual para los desiguales. Por el contrario, los desiguales requieren un trato desigual y ello justifica la intervención del Estado.

Una idea rectora de la intervención del Estado en la economía es la de la justicia social. Para Hayek se trata, sin embargo, de una idea sin sentido (Hayek, 1976a y 1978). A primera vista, muchos pueden encontrar este enunciado bastante chocante. Empero, se trata de un planteamiento que surge de su tesis principal acerca del carácter incompleto y descentralizado del conocimiento en la sociedad. Hayek sostiene que en sociedades donde prevalece el orden espontáneo del mercado, esto es, donde los individuos realizan libremente intercambios entre ellos, la posición que ocupa cada individuo en la sociedad es el resultado de esas múltiples interacciones y del azar. Independientemente del mérito y del esfuerzo de un individuo, las cosas le pueden salir mal. Por lo tanto, sería imposible establecer un criterio o principio que sirviera de guía para equilibrar la situación de todos esos individuos. 
La idea de lograr un equilibrio semejante es un espejismo en parte nutrido por el tipo de moralidad existente en las sociedades tradicionales. En éstas, el orden social es más simple porque el número y tipo de interacciones es mucho más limitado. El orden se puede mantener mediante el apego a un conjunto de deberes que sirven para encuadrar a las personas en roles nítidamente delimitados. De este encuadramiento surge la idea de que toda la sociedad puede ser ordenada de acuerdo a unos principios de justicia. En las sociedades de mercado, sostiene Hayek, la moralidad es abstracta. Depende de las reglas anónimas que han surgido en el curso de los intercambios que realizan los individuos, así como de la garantía de igualdad ante la ley que previene los favoritismos, la corrupción y el abuso. Imponer otros deberes equivaldría a distorsionar la orientación básica de los individuos en el mercado.

No obstante, en numerosos pasajes de su obra, Hayek defiende la idea de establecer instituciones que le garanticen un ingreso mínimo a los más desventajados. Se trata de una idea que justifica en términos de solidaridad, mas no de justicia. En una sociedad en la cual las interacciones de los individuos son múltiples y muy diversas, es imposible postular que una cierta distribución de recursos sea más o menos justa. Puesto que cada distribución es el resultado de un conjunto bastante complejo de interacciones, solamente por la vía de una reducción autoritaria podría uno poner en cuestión qué tan justa es. Esto no quiere decir que se deba ignorar la suerte de los más desventajados sino que la atención que se les prodigue no puede ser justificada en términos de justicia social.

Este planteamiento es, valga la pena subrayarlo, muy distinto del de muchos neoliberales que se identifican con Hayek pero que más bien merecerían el título de libertarios capitalistas. De una forma más o menos consciente, reiteran la denuncia hecha por Robert Nozick (1974) según la cual toda forma de asistencia es un hurto disfrazado de política social. Creo que Hayek nunca habría permitido ser reclutado en este bando. Lo que Hayek siempre defendió es que no hay alternativas al mercado y que el mercado genera desigualdades. Puesto que no hay criterio alguno que nos permitiera juzgar esas desigualdades y reducirlas de acuerdo con un principio de justicia, lo más que podría hacerse es proteger a los más desventajados de la adversidad.

A este respecto, sin embargo, la teoría social y política de Hayek adolece de una fuerte inconsistencia. Hayek era de la opinión de que los beneficios derivados de una mayor capacidad para producir más y más barato compensaban la reducción del número de agentes independientes en el mercado. El consiguiente aumento de tamaño y de poder de las empresas no sería un problema siempre y cuando hubiese suficientes empresas compitiendo entre sí. Esta competencia sería, con respecto al mercado, un mecanismo de regulación de regulación interno mucho más efectivo que cualquier supervisión y control externos ejercidos por el Estado. Hayek desdeñó el riesgo de la cartelización, esto es, la suscripción de acuerdos formales e informales hechos entre las grandes empresas para 
poner barreras de entrada y dividir el mercado entre los productores, limitar la oferta y fijar los precios. Si este fenómeno lo consideramos en tándem con su descripción del proceso democrático, entonces la efectividad de su propuesta de una red social de apoyo a los más desventajados queda completamente en entredicho.

En efecto, el funcionamiento de esa red depende del proceso democrático. Incluso si sus principios básicos se sustrajeran de la discusión ordinaria de las asambleas representativas mediante el expediente de constitucionalizar su vigencia, esas asambleas siempre tendrían que intervenir, como en efecto lo hacen, para fijar las políticas y asignar los recursos necesarios para su funcionamiento. Puesto que el proceso de deliberación democrática es sesgado por la influencia de grupos organizados de interés, habríamos de observar que en ese proceso de deliberación muchos grupos poderosos intervendrían para reducir a su mínima expresión la mencionada red social de apoyo puesto que esa sería una forma efectiva de aliviar su carga tributaria. Las discusiones acerca de los recortes del llamado gasto público social en numerosos países (Estados Unidos, Reino Unido, etc.) ponen en evidencia este punto flaco de la teoría de Hayek: contrario a lo que él pensaba, esa teoría no postula ningún mecanismo para que la red social de apoyo a los más vulnerables sea realmente efectiva.

\section{Críticas a Hayek desde la izquierda y desde la derecha}

Dado que la obra de Hayek abarca numerosos aspectos, no es de extrañar que sus planteamientos hayan recibido numerosas críticas. Aquí me voy a concentrar en algunas de ellas, especialmente, en las dirigidas contra su argumento epistemológico contra el socialismo, su rechazo a la idea de justicia social y su idea de la democracia. Podemos decir que estas son las críticas a Hayek desde la izquierda. Desde la derecha Hayek también ha recibido críticas que bien vale la pena reseñar aquí. Soy de los que cree que tenemos mucho que aprender de una conversación con el pensamiento conservador.

Basado en una cuidadosa lectura de la filosofía de Ludwig Wittgenstein, Nigel Pleasants (1997 y 1999) arguye que no puede haber nada como un conocimiento privado. El conocimiento implica una práctica social en la cual todas las afirmaciones que conllevan una pretensión de verdad están siempre abiertas a la crítica y el escrutinio. Para decirlo en un lenguaje popperiano, en el ámbito del conocimiento, toda afirmación debe admitir ser falseada, esto es, probada falsa en su confrontación con los hechos. Este es un punto en el cual converge un economista liberal como Kenneth Arrow (1994), pues él ha mostrado que el individualismo metodológico es muy poco adecuado para explicar la producción y circulación social del conocimiento.

La crítica de Pleasants tiene varias implicaciones políticas. En primer lugar, Pleasants destaca que la idea de conocimiento privado en Hayek le da a los deseos personales una investidura que no merecen. En efecto, al otorgarles el aura de conocimiento, las preferencias 
individuales se transforman en proposiciones que se tornan reacias a toda demanda pública de rendición de cuentas. Dicho de un modo más sencillo, ¿̇por qué hemos de creer al pie de la letra en lo que un individuo cree saber acerca del mundo e incluso acerca de sí mismo? Nuestra percepción del mundo está sujeta a numerosos errores. Podemos equivocarnos incluso acerca de nosotros mismos. El punto de Pleasants consiste justamente en que, privados del espacio público en el cual validamos nuestras creencias, el conocimiento individual muy pronto se transforma en la tiranía de las preferencias individuales.

En concordancia con lo anterior, Pleasants critica a Hayek por confundir la rentabilidad del productor individual con la eficiencia del conjunto. El elogio que Hayek hace del sistema de precios como sistema que permite la interacción descentralizada de muchos individuos soslaya uno de los problemas más graves del capitalismo: lo que tiene sentido al nivel de los productores individuales puede, sin embargo, como acontece en la actualidad, conducir al agotamiento global de recursos escasos e irreparables y, finalmente, al empobrecimiento de todos los individuos. El calentamiento global es el mejor testimonio de los límites del pensamiento de Hayek acerca de las virtudes del conocimiento privado. El mercado no ha logrado darle un peso adecuado a la forma como usamos los recursos naturales. Ha sido mediante la legislación que se han impuesto límites a la extracción y explotación de recursos, límites que procuran que los productores internalicen costos que, de otro modo, se los trasladarían a la sociedad. La resistencia de poderosos grupos privados contra esos límites es un testimonio adicional de cuán poco conocimiento tienen muchos individuos del daño que le hacen a la naturaleza, a otros individuos y a sí mismos, y de lo mal que circula el conocimiento de ese daño en el mercado.

Además, puede decirse que Hayek subestimó la capacidad de diferentes instituciones para agregar el conocimiento social. En efecto, muchas empresas toman sus decisiones luego de recaudar un gran volumen de información acerca de las necesidades, los gustos y los hábitos de los consumidores. Si esta actividad la pueden llevar a cabo agentes económicos privados, ¿qué le impide hacerla al Estado con el fin de asignar de una forma mejor los limitados recursos del presupuesto público? La crítica de Hayek en contra de una economía planificada centralmente no nos debería llevar a creer que no es posible ninguna clase de agregación del conocimiento social ni a negar el beneficio que la sociedad en su conjunto podría adquirir del uso de ese conocimiento.

Los argumentos de Hayek en favor del conocimiento privado y del orden espontáneo tampoco deberían tomarse al pie de la letra en lo que respecta a la total carencia de significado del concepto de justicia social. Su punto acerca de la imposibilidad de ajustar la distribución de recursos en una sociedad compleja supone que la evaluación de esa distribución la realizamos siempre desde el punto de vista de los resultados. Dicho de otro modo, la crítica de Hayek al concepto de justicia social parte de la premisa que la justicia social consiste en igualar la suma de recursos correspondiente a cada individuo. 


\section{Juan Gabriel Gómez Albarello}

Esta no es la única forma de ver las cosas. En vez de concentrarnos en el punto de llegada, esto es, en la cantidad de recursos que cada individuo ha logrado acumular, podemos concentrarnos en el punto de partida, es decir, en los recursos con que contaba cada individuo para realizar su proyecto de vida al momento de adquirir las capacidades para llevarlo a cabo. Si nos tomamos en serio la idea de que todos los individuos disfruten de una libertad igual, entonces, para ser consecuentes con esta premisa deberíamos evaluar la forma en que diferencias no atribuibles al esfuerzo individual impactan negativamente en la capacidad que tiene cada individuo para lograr una vida plena.

En efecto, hay un buen número de diferencias que son independientes de la acción de los individuos. Algunas de esas diferencias son atribuibles a la lotería de la naturaleza así como a la lotería de la sociedad. Haríamos bien en admitir que haber nacido en una familia con más o menos recursos y dotado de más o menos habilidades es una contingencia respecto de la cual nadie tiene ninguna responsabilidad. Sin embargo, si no realizamos ningún esfuerzo por igualar a los individuos en el punto de partida, esto es, en lo que respecta a las oportunidades para realizar sus capacidades, estaríamos en la posición de quien los tratara a todos como si fueran responsables de haber escogido dónde nacieron y con qué capacidades.

Este es el quid de las teorías de justicia distributiva basadas en el principio de igualdad de oportunidades. Lo que estas teorías hacen es evaluar negativamente las situaciones sociales que hacen que algunos individuos puedan desarrollar al máximo sus potencialidades, mientras que otros ven esas potencialidades reducidas a su mínima expresión. No sólo eso. Lo que esas teorías procuran es brindarnos indicaciones acerca de la forma en que deberíamos distribuir nuestros recursos para asegurar una igualdad de oportunidades a todos los individuos.

Lo que estas teorías nos plantean es que deberíamos rechazar distribuciones de recursos que no dependen del esfuerzo individual sino de privilegios de clase o condición previos a ese esfuerzo. Se trata de privilegios que surgen de estructuras desiguales de oportunidades que limitan lo que un individuo puede obtener mediante el cultivo de sus propias capacidades y el despliegue de su propia actividad. Vistas así las cosas, la idea de justicia social recupera todo su sentido. Una demanda de justicia al nivel global de las instituciones sociales demanda una revisión global que le permita a cada uno vivir su vida libremente, no como un destino impuesto. Los aportes de Ronald Dworkin (2002) y John Roemer (1998) proporcionan a este respecto un contrapunto muy fundamentado a la crítica de Hayek a la idea de justicia social.

Los planteamientos de Hayek acerca de la justicia social son susceptibles a otro tipo de crítica: al oponer el modelo de un orden espontáneo al de uno construido racionalmente, Hayek soslaya el hecho de que muchos conflictos distributivos son abordados y resueltos en el marco de discusiones morales acerca de la justificación que tienen las 
instituciones económicas y políticas. Estas discusiones constituyen la sustancia de la vida política de las sociedades modernas en las cuales el balance entre valores en competencia se pone de presente en la solución de muchos problemas particulares.

A primera vista, estos parecen ser los argumentos de un defensor de la concepción republicana de la democracia. Sin embargo, se trata de un argumento articulado por Frank Knight (Emmett, 2007), el mentor intelectual de los economistas que luego serían reconocidos como miembros de la Escuela de Chicago. Ser libre no sólo es tener la posibilidad de realizar intercambios sin la interferencia de una autoridad externa, es también tener la de discutir acerca de lo que es justo y lo que es injusto.

En este mismo orden de ideas, la crítica de Hayek a la democracia también deberíamos tomarla como un grano de sal. Aunque su descripción del proceso democrático "realmente existente" es acertada, creo que esta crítica no agota todo el sentido que la democracia sigue teniendo. Hayek no valoró adecuadamente la conexión intrínseca que existe entre la democracia y el imperio de la ley. Si bien la tensión entre la democracia y el Estado de derecho es permanente, es difícil creer que el Estado de derecho pueda mantenerse sin la democracia. La oposición entre el gobierno de los hombres y el gobierno de la ley captura muy bien la oposición entre la arbitrariedad despótica y la garantía de una ley general basada en los derechos individuales. Sin embargo, en última instancia, el imperio del derecho depende de que haya gente que ponga en marcha la maquinaria del Estado para hacer cumplir la ley y hacer efectivos sus derechos. Donde no hay un acuerdo acerca de valores básicos y la participación política es limitada, es muy probable que el cumplimiento de la ley siempre se haga en una forma sesgada. Entre nosotros, el dicho "la ley es para los de ruana" no puede considerarse independiente de un proceso político en el cual hay muchos individuos y grupos de individuos desempoderados, esto es, carentes de la capacidad de poner en cuestión la aplicación selectiva de la ley mediante demandas de rendición de cuentas.

Jeremy Waldron (2009) ha articulado este punto muy bien en su revisión sobre el significado del constitucionalismo. Sin embargo, no es aventurado decir que su contribución es deudora del análisis de Jürgen Habermas (1998) acerca de la interdependencia de la democracia y el Estado de derecho. Se trata de una interdependencia que concierne no solamente al terreno de la práctica política sino también a la manera en la cual comprendemos esta práctica. Uno de los aportes de Habermas ha consistido en mostrar que la idea liberal de la libertad como ausencia de restricciones externas es insuficiente y precisa de la idea de libertad como auto-legislación. Quizá la mejor evidencia de esta conexión interna entre los sentidos negativo y positivo de libertad es la expansión de los poderes de vigilancia del Estado allí donde casi la mitad de la población no participa en el proceso político: en los Estados Unidos.

Esto no quiere decir que aquí se agote nuestra indagación acerca de la libertad. Habermas, al igual que Hayek, suponen un concepto no problemático de sujeto. Luego de 


\section{Juan Gabriel Gómez Albarello}

extraordinarios descubrimientos geográficos, maravillosas invenciones y una minuciosa comprensión de procesos externos, los seres humanos parecemos haber redescubierto el terreno interior en un regreso a la pregunta, ¿quiénes somos? La trascendencia de esta pregunta seguramente ha de alterar el significado que le atribuimos a la democracia.

Por otro lado, desde la derecha la crítica conservadora a Hayek ha consistido en poner el énfasis en un supuesto de su razonamiento y en acusar el efecto que sobre el mismo tiene la economía de mercado: el lazo social o, dicho de otro modo, la membresía en una comunidad política concreta. El pensador conservador Roger Scruton (2007) cuestiona a Hayek por no darle una adecuada consideración al efecto corrosivo que tiene el mercado sobre el vínculo social. ${ }^{10}$ Donde todo entra en el mercado, incluidas todas aquellas cosas que consideramos tabú, en otras palabras, donde todo está a la venta y donde hay un comprador para todo, el vínculo social termina por disolverse. Antes que él, el sociólogo Max Weber (1968) ya había advertido que los lazos basados en el interés individual no son duraderos. Se necesita que los individuos sostengan muchas instituciones, distintas del mercado, que contribuyan al mantenimiento del orden social.

Un tópico fundamental del pensamiento conservador es la importancia de la diferencia entre próximos y extraños, una distinción que a su turno evoca otra: la de amigos y enemigos. De hecho, la piedra de toque de la membresía en una comunidad política pareciera ser la enemistad. Es la necesidad de combatir al enemigo lo que motiva a los miembros de una comunidad a movilizarse en su defensa y, en ese proceso, a apelar a un sentido de lealtad que no puede ser reducido a los cálculos de utilidad propios de la sociedad de mercado. El diagnóstico conservador sería que una comunidad en la cual la apelación a ese sentido de lealtad caiga en el vacío está condenada a desaparecer. El argumento conservador es utilizado con mayor frecuencia en relación con los enemigos externos, pero también se extiende a la dimensión interna en temas que en la actualidad han llegado a superponerse como la lucha contra el terrorismo y los desafíos de la multiculturalidad. Entre nosotros, el uribismo es un ejemplo de un tipo de pensamiento conservador pero carismático puesto que la pertenencia a la nación es establecida por referencia a la lealtad a un líder y a su proyecto de derrotar al enemigo interno.

La crítica del pensamiento conservador a Hayek debe tomarse en serio. En lo que respecta a los límites del mercado, esta línea de cuestionamiento se encuentra con las críticas socialistas. Ambas tradiciones, a pesar de sus diferencias, comparten la idea de que el mercado no es un orden que se auto-genera, este depende de un orden social previo al

\footnotetext{
${ }^{10}$ Con ayuda de modelos formales, Walter J. Schultz (2001) ha mostrado que, en ausencia de restricciones morales, un conjunto de individuos egoístas no podrían alcanzar una óptima asignación de recursos mediante intercambios en el mercado. El punto de Schultz es también que el mercado por sí mismo no puede generar esas restricciones.
} 
cual ese mercado puede destruir si no se le limita. El énfasis en el peso de la membresía en el mantenimiento del orden social obliga a todo pensamiento cosmopolita a responder cómo podría sostenerse ese orden si se ablandan las diferencias entre próximos y extraños. Lo que ocurre hoy en la Unión Europea es una buena demostración de que una mera unión económica, basada en una sola moneda, no es suficiente para integrar en una misma unidad política a millones de personas que se sienten vinculadas a tradiciones históricas muy distintas. Los cosmopolitas podrían replicar, sin embargo, que la Unión Europea es el peor ejemplo de cosmopolitismo precisamente porque se trata de una unidad en la que el sentido primario de comunidad nunca se reemplazó adecuadamente - en el corazón de cada ciudadano europeo palpita más fuerte la lealtad a su nación que a la de la Unión- y en la que la participación democrática quedó severamente limitada en favor de la centralización del gobierno económico. El mismo Hayek, en su trabajo sobre la desnacionalización de la moneda, previó que un circulante único para Europa podría significar más tiranía, no menos. Con relación a este último punto, los hechos le han dado la razón (Sen, 2012).

\section{Conclusión}

El pensamiento político contemporáneo se las ve una y otra vez con la tensión entre igualdad política y desigualdad económica. El intento por resolver esta tensión se me figura análogo al de los esfuerzos por resolver la cuadratura del círculo. La teoría de la justicia de John Rawls y los modelos de democracia deliberativa pueden ser vistos desde este prisma. La obra de Friedrich von Hayek ofrece el contrapunto más elocuente y profundo a esos intentos de solución de la aludida tensión.

Hayek nos ha legado una obra en la cual la clave reside en la imposibilidad de agregar el conocimiento privado y en la importancia de mantener instituciones que permitan la continua regeneración de un orden social espontáneo. De este planteamiento Hayek extrae numerosas implicaciones cuyo común denominador es ponerle cortapisas al despotismo político. En mi opinión, aquí radican también las principales limitaciones de Hayek: no supo darle un peso adecuado al despotismo económico ni al efecto que tiene sobre la naturaleza la continua externalización de los costos ambientales de la actividad económica. Ambos factores demandan una intervención política en el mercado, que ha de llevarse a cabo tanto en la esfera de local, como en la nacional y la internacional. Prueba de ello son la creciente desigualdad económica y la destrucción de nuestro medio ambiente.

\section{Referencias Bibliográficas}

Arrow, K. J. (1994). "Methodological Individualism and Social Knowledge", American Economic Review, 84 (2), 1-9.

Bobbio, N. (1988). Liberalismo e democracia. São Paulo: Editora Brasiliense. 
Brass, T. (2011). Labour Regime Change in the Twenty-First Century: Unfreedom, Capitalism and Primitive Accumulation. Leiden: Brill.

Buchanan, J, M. y Tullock, G. (1962). The Calculus of Consent, Logical Foundations of Constitutional Democracy. Ann Arbor: University of Michigan Press.

Bukharin, N. (1982). Selected Writings on the State and the Transition to Socialism. Day, R. B. (Ed.). Nueva York: M. E. Sharpe.

Davis, K., Schoen, C., Schoenbaum, S. C., Doty, M. M., Holmgren A. L., Kriss J. L., y Shea K. K. (2007). Mirror, Mirror on The Wall: An International Update on the Comparative Performance of American Health Care. Commonwealth Fund. Disponible en: http://www.commonwealthfund.org/Publications/FundReports/2007/May/Mirror--Mirror-on-the-Wall--An-International-Update-onthe-Comparative-Performance-of-American-Healt.aspx.

Dobb, M. (1928). Russian Economic Development since the Revolution. Nueva York: E. P. Dutton \& Co.

Dobb, M. (1955). On Economic Theory and Socialism. Londres: Routledge.

Dowlah, A. y Elliot, J. (1997). The Life and Times of Soviet Socialism. Westport: Praeger.

Downs, A. (1957). An Economic Theory of Democracy. Nueva York: Harper.

Dworkin, R. (2002). Sovereign Virtue. Cambridge: Harvard University Press.

Emmett, R. B. (2007). “Knight's Challenge (to Hayek): Spontaneous Order is not Enough for Governing a Liberal Society”. En: L. Hunt y P. McNamara (Eds), Liberalism, Conservatism, and Hayek's Idea of Spontaneous Order (pp. 67-86). Nueva York: Palgrave.

Engels, F. (1977). La Subversión de la Ciencia por el Señor Eugen Dühring (AntiDühring). Barcelona: Crítica.

Friedman, M. y R. Friedman. (1980). Free to Choose: A Personal Statement. San Diego: Harcourt.

Gesell, S. (1958). The Natural Economic Order. Londres: Peter Owen Ltd.

Habermas, J. (1998). Facticidad y Validez: Sobre el Derecho y el Estado Democrático de Derecho en términos de Teoría del Discurso. Madrid: Trotta.

Hanson, P. (2003). The Rise and Fall of the Soviet Economy: An Economic History of the USSR from 1945. Londres: Longman.

Hayek, F. (1945). The Use of Knowledge in Society. American Economic Review, 35 (4), 519-30. 
Hayek, F. (1948). Individualism and Economic Order. Chicago: University of Chicago Press.

Hayek, F. (1952). Scientism and the Study of Society. Glencoe: Free Press.

Hayek, F. (1960). “The Intellectuals and Socialism”. En: G. B. Huszard (Ed.), The Intellectuals: A Controversial Portrait (pp. 371-384). Glencoe: Free Press

Hayek, F. (1960). The Constitution of Liberty. Chicago: Chicago University Press.

Hayek, F. (1967). “The Theory of Complex Phenomena”. En: F. Hayek (Ed.). Studies in Philosophy, Politics and Economics (pp. 22-42). Londres: Routledge \& Kegan Paul.

Hayek, F. (1973). Law, Legislation and Liberty: A New Statement of the Liberal Principles of Justice and Political Economy. 1: Rules and Order. Londres: Routledge \& Kegan Paul.

Hayek, F. (10 de diciembre de 1974). Friedrich von Hayek's Speech at the Nobel Banquet (Estocolmo, Suecia). Disponible en: http://www.nobelprize.org/nobel_prizes/ economic-sciences/laureates/1974/hayek-speech.html

Hayek, F. (1975). The Pretence of Knowledge. Swedish Journal of Economics, 77 (4), 433-442.

Hayek, F. (1976a). Law, Legislation and Liberty: A New Statement of the Liberal Principles of Justice and Political Economy. 2: The Mirage of Social Justice. Londres: Routledge \& Kegan Paul.

Hayek, F. (1976b). The Denationalization of Money. Londres: Institute of Economic Affairs.

Hayek, F. (1978). The Atavism of Social Justice. En: F. Hayek (Ed.). New Studies in Philosophy, Politics, Economics and the History of Ideas (pp. 57-68). Londres: Routledge.

Hayek, F. (1979). Law, Legislation and Liberty: A New Statement of the Liberal Principles of Justice and Political Economy. 3: The Political Order of a Free People. Londres: Routledge \& Kegan Paul.

Hayek, F. (2006). The Road to Serfdom. Londres: Routledge.

Hook, S. (1968). The Enlightenment and Marxism. Journal of the History of Ideas, 29 (1), 93-108.

Kolakowski, L. (1978). Main Currents of Marxism: Its Origins, Growth and Dissolution. 1: The Founders. Oxford: Oxford University Press.

Lange, O. (1936). “On the Economic Theory of Socialism, Part One”, Review of Economic Studies, 4 (1), 53-71. 
Lange, O. (1937). "On the Economic Theory of Socialism, Part Two", Review of Economic Studies, 4 (2), 123-142.

Lenin, V. I. (1993). El Estado y la Revolución. Madrid: Planeta.

Lerner, A. (1934). "Economic Theory and the Problems of a Socialist Economy". Review of Economic Studies, 2 (1), 51-61.

Lerner, A. (1936). “A Note on Socialist Economics”. Review of Economic Studies, 4 (1), 72-76.

Lerner, A. (1937). "Statics and Dynamics in Socialist Economics". Economic Journal, 47 (186), 253-270.

Luxemburg, R. (1969). La Revolución Rusa. Barcelona: Anagrama.

Marx, K. (1998). Manifiesto Comunista. Barcelona: Crítica.

Marx, K. (2000). El Capital. 1: El Proceso de Producción del Capital. México: Fondo de Cultura Económica.

Mises, L. v. (1951). Socialism: An Economic and Sociological Analysis. New Haven: Yale University Press.

Neurath, O. (2004). Economic Writings; Selections 1904-1945. Uebel, T. E. y Cohen, R. S. (Eds.). Dordrecht: Kluwer.

North, D. (2004). Understanding the Process of Economic Change. Princeton: Princeton University Press.

Nozick, R. (1974). Anarchy, State, and Utopia. Nueva York: Basic Books.

Petsoulas, C. (2001). Hayek's Liberalism and Its Origins: His Idea of Spontaneous Order and the Scottish Enlightenment. Londres: Routledge.

Pleasants, N. (1997). The Epistemological Argument against Socialism: A Wittgensteinian Critique of Hayek and Giddens. Inquiry, 40, 23-45.

Pleasants, N. (1999). Wittgenstein and the Idea of Critical Social Theory. Londres: Routledge.

Polanyi, K. (2001). The Great Transformation: The Political and Economic Origins of Our Time. Boston: Beacon Press.

Poulantzas, N. (1979). Estado, Poder y Socialismo. México: Siglo XXI Editores.

Riker, W. H. (1982). Liberalism against Populism: A Confrontation between the Theory of Democracy and the Theory of Social Science. Long Grove: Waveland Press.

Roemer, J. (1998). Equality of Opportunity. Cambridge: Harvard University Press. 
Schultz, W. J. (2001). The Moral Conditions of Economic Efficiency. Nueva York: Cambridge University Press.

Scruton, R. (2007). "Hayek and Conservatism”. En: E. Feser (Ed.), Cambridge Companion to Hayek (pp. 208-231). Nueva York: Cambridge University Press.

Sen, A. (22 de mayo de 2012). The Crisis of European Democracy. New York Times. Disponible en: http,//www.nytimes.com/2012/05/23/opinion/the-crisis-ofeuropean-democracy.html?_r=o.

Smith, Vernon. (2005). Hayek and Experimental Economics. Review of Austrian Economics, 18 (2), 135-144.

Stedman Jones, D. (2012). Masters of the Universe: Hayek, Friedman, and the Birth of Neoliberal Politics. Princeton: Princeton University Press.

Waldron, J. (2009). “Constitutionalism - A Skeptical View”. En: T. Christiano y J. Christman (Eds), Contemporary Debates in Political Philosophy (pp. 267-282). Nueva York: Blackwell.

Weber, M. 1968. Economy and Society. An Outline of Interpretive Sociology. New York: Bedminster Press.

Williamson, Oliver. 2000. The New Institutional Economics: Taking Stock and Looking Ahead. Journal of Economic Literature, 38 (3), 595-613.

Wittman, D. A. (1995). The Myth of Democratic Failure. Chicago: Chicago University Press. 
